\title{
CUBIC GRAPHS WITH APPLICATION
}

\author{
SHEIKH RASHID ${ }^{1}$, NAVEED YAQOOB ${ }^{2, *}$, MUHAMMAD AKRAM ${ }^{3}$, MUHAMMAD $^{-}$ \\ GULISTAN $^{1}$ \\ ${ }^{1}$ Department of Mathematics, Hazara University, Mansehra, Pakistan \\ ${ }^{2}$ Department of Mathematics, College of Science Al-Zulf, Majmaah University, Al-Zulf, Saudi Arabia \\ ${ }^{3}$ Department of Mathematics, University of the Punjab, New Campus, Lahore, Pakistan \\ ${ }^{*}$ Corresponding author: nayaqoob@ymail.com
}

\begin{abstract}
We introduce certain concepts, including cubic graphs, internal cubic graphs, external cubic graphs, and illustrate these concepts by examples. We deal with fundamental operations, Cartesian product, composition, union and join of cubic graphs. We discuss some results of internal cubic graphs and external cubic graphs. We also describe an application of cubic graphs.
\end{abstract}

\section{IntRoduction}

Cubic sets are one of the real generalizations of fuzzy sets [27] provided by Jun et al. [9-11,15,26] during the last five years. They developed cubic set theory in many directions and for more detail about cubic sets one can see [12]. Kang and Kim [13] studied mappings of cubic sets. Muhiuddin et al. [18] presented the idea of stable cubic sets.

Fuzzy graphs were studied by Rosenfeld [23] and give a few theoretical ideas in spite of the fact that the fundamental thought was presented by Kauffmann [14] in 1973. Bhattacharya [6] gave some remarks on fuzzy graphs. A book written by Mordeson and Nair [17] is devoted especially to the study of fuzzy graphs

Received 2018-03-13; accepted 2018-05-22; published 2018-09-05.

2010 Mathematics Subject Classification. 68R10, 05C72.

Key words and phrases. cubic sets; cubic graphs; internal and external cubic graphs.

(C)2018 Authors retain the copyrights of their papers, and all open access articles are distributed under the terms of the Creative Commons Attribution License. 
and fuzzy hypergraphs. Akram et al. gave the idea of interval-valued fuzzy graphs [1,2], intuitionistic fuzzy graphs [3] and bipolar fuzzy graphs [4,5]. Borzooei and Rashmanlou [7] studied Cayley interval-valued fuzzy threshold graphs. Buckley [8] introduced self-centered graphs. Sunitha et al. [25] characterized g-self centered fuzzy graphs. Mishra et al. [16] studied coherent category of interval-valued intuitionistic fuzzy graphs. Pal et al. [19] and Pramanik et al. [21,22] added some useful results to the theory of interval-valued fuzzy graphs. Parvathi et al. [20] provided some different operations on intuitionistic fuzzy graphs and Sahoo and Pal [24] studied product of intuitionistic fuzzy graphs.

In this paper we study some operations on cubic graphs. Internal and external cubic graphs are studied with some example. We provided some conditions for union and join of external and internal cubic graphs.

\section{Preliminaries}

Here we recall some basic helping material from the existing literature.

Definition 2.1. A graph is denoted by $\Omega^{*}=(P, Q)$, where $P$ denotes the set of vertices of $\Omega^{*}$ and $Q$ stands for the set of edges of $\Omega^{*}$.

Definition 2.2. [12] Let $T$ be a non-empty set. By a cubic set in $T$ we mean a structure

$$
\Lambda=\left\{\left\langle t, \widetilde{\varpi}_{\Lambda}(t), \mu_{\Lambda}(t)\right\rangle \mid t \in T\right\}
$$

in which $\widetilde{\varpi}_{\Lambda}$ is an interval-valued fuzzy set in $T$ and $\mu_{\Lambda}$ is a fuzzy set in $T$.

A cubic set $\Lambda=\left\{\left\langle t, \widetilde{\varpi}_{\Lambda}(t), \mu_{\Lambda}(t)\right\rangle \mid t \in T\right\}$ is simply denoted by $\Lambda=\left\langle\widetilde{\varpi}_{\Lambda}, \mu_{\Lambda}\right\rangle$.

Definition 2.3. [12] Let $T$ be a non-empty set. A cubic set $\Lambda=\left\langle\widetilde{\varpi}_{\Lambda}, \mu_{\Lambda}\right\rangle$ in $T$ is said to be an internal cubic (resp., external cubic) set if

$$
\varpi_{\Lambda}^{-}(t) \leq \mu_{\Lambda}(t) \leq \varpi_{\Lambda}^{+}(t) \quad\left(\text { resp. }, \mu_{\Lambda}(t) \notin\left(\varpi_{\Lambda}^{-}(t), \varpi_{\Lambda}^{+}(t)\right)\right)
$$

for all $t \in T$.

Definition 2.4. [12] For any $\Lambda_{i}=\left\{\left\langle t, \widetilde{\varpi}_{\Lambda_{i}}(t), \mu_{\Lambda_{i}}(t)\right\rangle \mid t \in T\right\}$ where $i \in I$, we define

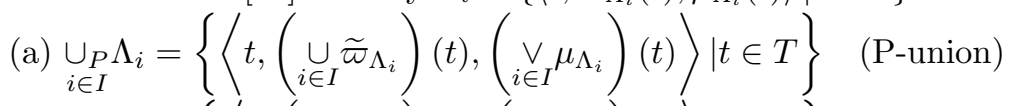

(b) $\cup_{i \in I} \Lambda_{i}=\left\{\left\langle t,\left(\underset{i \in I}{\cup} \widetilde{\varpi}_{\Lambda_{i}}\right)(t),\left(\underset{i \in I}{\wedge} \mu_{\Lambda_{i}}\right)(t)\right\rangle \mid t \in T\right\} \quad$ (R-union)

\section{CUBIC GRAPHS}

We develop the theory of a cubic graph and some operations on cubic graph. 
Definition 3.1. Let $M^{*}=\langle P, Q\rangle$ be a graph. A cubic graph of a graph $M^{*}=\langle P, Q\rangle$, is the structure $M=\langle\alpha, \beta\rangle$, where $\alpha=\left\langle\widetilde{\varpi}_{\alpha}, \mu_{\alpha}\right\rangle$ is the cubic set representation for the vertex $P$ and $\beta=\left\langle\widetilde{\varpi}_{\beta}, \mu_{\beta}\right\rangle$ denotes the cubic set representation for the edge $Q$, with

$$
\begin{aligned}
\widetilde{\varpi}_{\alpha}: & P \rightarrow D[0,1], \mu_{\alpha}: P \rightarrow[0,1], \\
\text { and } \widetilde{\varpi}_{\beta}: & Q \rightarrow D[0,1], \mu_{\beta}: Q \rightarrow[0,1],
\end{aligned}
$$

such that

$$
\begin{aligned}
\widetilde{\varpi}_{\beta}\left(p_{i} p_{j}\right) & \preceq r \min \left\{\widetilde{\varpi}_{\alpha}\left(p_{i}\right), \widetilde{\varpi}_{\alpha}\left(p_{j}\right)\right\}, \\
\mu_{\beta}\left(p_{i} p_{j}\right) & \leq \max \left\{\mu_{\alpha}\left(p_{i}\right), \mu_{\alpha}\left(p_{j}\right)\right\},
\end{aligned}
$$

for all $\left(p_{i}, p_{j}\right) \in Q \subseteq P \times P$.

Example 3.1. Let us consider a graph $\Omega^{*}=(P, Q)$ such that $P=\left\{p_{1}, p_{2}, p_{3}, p_{4}\right\}, Q=\left\{p_{1} p_{2}, p_{2} p_{3}, p_{3} p_{4}, p_{4} p_{1}\right\}$. Let $\alpha$ be a cubic set of $P$ and let $\beta$ be a cubic set of $Q$ defined by

\begin{tabular}{c||c|c}
\hline$P$ & $\widetilde{\varpi}_{\alpha}$ & $\mu_{\alpha}$ \\
\hline \hline$p_{1}$ & {$[0.1,0.5]$} & 0.7 \\
\hline$p_{2}$ & {$[0.3,0.7]$} & 0.2 \\
\hline$p_{3}$ & {$[0.2,0.4]$} & 0.2 \\
\hline$p_{4}$ & {$[0.1,0.8]$} & 0.7 \\
\hline
\end{tabular}

\begin{tabular}{c||c|c}
\hline$Q$ & $\widetilde{\varpi}_{\beta}$ & $\mu_{\beta}$ \\
\hline \hline$p_{1} p_{2}$ & {$[0.1,0.4]$} & 0.4 \\
\hline$p_{2} p_{3}$ & {$[0.1,0.3]$} & 0.1 \\
\hline$p_{3} p_{4}$ & {$[0.1,0.4]$} & 0.5 \\
\hline$p_{4} p_{1}$ & {$[0.1,0.4]$} & 0.3 \\
\hline
\end{tabular}

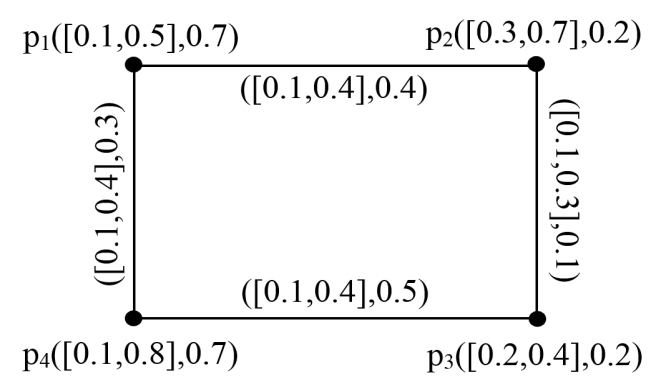

Figure 1. Cubic graph

By routine calculations, it can be observed that the graph shown in Fig. 1 is a cubic graph.

Example 3.2. Consider a graph $\Omega^{*}=(P, Q)$. Let $\alpha$ be a cubic set of $P$ and let $\beta$ be a cubic set of $Q$ defined by

$$
\mu_{\alpha}\left(p_{i}\right)=\frac{\varpi_{\alpha}^{-}\left(p_{i}\right)+\varpi_{\alpha}^{+}\left(p_{i}\right)}{2} \text { and } \mu_{\beta}\left(e_{i}\right)=\frac{\varpi_{\beta}^{-}\left(e_{i}\right)+\varpi_{\beta}^{+}\left(e_{i}\right)}{2} .
$$

Then $M=\langle\alpha, \beta\rangle$ is a cubic graph of $\Omega^{*}$. 
Remark 3.1. If $\widetilde{\varpi}_{\beta}\left(p_{i} p_{j}\right)=[0,0]$ and $\mu_{\beta}\left(p_{i} p_{j}\right)=0$, then the cubic graph $M=\langle\alpha, \beta\rangle$ has no edge.

Definition 3.2. Let $M_{1}=\left\langle\alpha_{1}, \beta_{1}\right\rangle$ and $M_{2}=\left\langle\alpha_{2}, \beta_{2}\right\rangle$ be two cubic graphs of the graphs $\Omega_{1}^{*}$ and $\Omega_{2}^{*}$, respectively. The Cartesian product of $M_{1}$ and $M_{2}$ is denoted by $M_{1} \times M_{2}=\left\langle\alpha_{1} \times \alpha_{2}, \beta_{1} \times \beta_{2}\right\rangle$ and is defined as follows:

(i) $\left\{\begin{array}{c}\left(\widetilde{\varpi}_{\alpha_{1}} \times \widetilde{\varpi}_{\alpha_{2}}\right)\left(p_{1}, p_{2}\right)=r \min \left\{\widetilde{\varpi}_{\alpha_{1}}\left(p_{1}\right), \widetilde{\varpi}_{\alpha_{2}}\left(p_{2}\right)\right\} \\ \left(\mu_{\alpha_{1}} \times \mu_{\alpha_{2}}\right)\left(p_{1}, p_{2}\right)=\max \left\{\mu_{\alpha_{1}}\left(p_{1}\right), \mu_{\alpha_{2}}\left(p_{2}\right)\right\}\end{array}\right.$

for all $\left(p_{1}, p_{2}\right) \in P=P_{1} \times P_{2}$,

(ii) $\left\{\begin{array}{c}\left(\widetilde{\varpi}_{\beta_{1}} \times \widetilde{\varpi}_{\beta_{2}}\right)\left(\left(q, q_{2}\right)\left(q, p_{2}\right)\right)=r \min \left\{\widetilde{\varpi}_{\alpha_{1}}(q), \widetilde{\varpi}_{\beta_{2}}\left(q_{2} p_{2}\right)\right\} \\ \left(\mu_{\beta_{1}} \times \mu_{\beta_{2}}\right)\left(\left(q, q_{2}\right)\left(q, p_{2}\right)\right)=\max \left\{\mu_{\alpha_{1}}(q), \mu_{\beta_{2}}\left(q_{2} p_{2}\right)\right\}\end{array}\right.$

for all $q \in P_{1}$, and $q_{2} p_{2} \in Q_{2}$,

(iii) $\left\{\begin{array}{c}\left(\widetilde{\varpi}_{\beta_{1}} \times \widetilde{\varpi}_{\beta_{2}}\right)\left(\left(q_{1}, r\right)\left(p_{1}, r\right)\right)=r \min \left\{\widetilde{\varpi}_{\beta_{1}}\left(q_{1} p_{1}\right), \widetilde{\varpi}_{\alpha_{2}}(r)\right\} \\ \left(\mu_{\beta_{1}} \times \mu_{\beta_{2}}\right)\left(\left(q_{1}, r\right)\left(p_{1}, r\right)\right)=\max \left\{\mu_{\beta_{1}}\left(q_{1} p_{1}\right), \mu_{\alpha_{2}}(r)\right\}\end{array}\right.$

for all $r \in P_{2}$, and $q_{1} p_{1} \in Q_{1}$.

Example 3.3. Consider two cubic graphs $M_{1}=\left\langle\alpha_{1}, \beta_{1}\right\rangle$ and $M_{2}=\left\langle\alpha_{2}, \beta_{2}\right\rangle$ as shown in figure 2 .

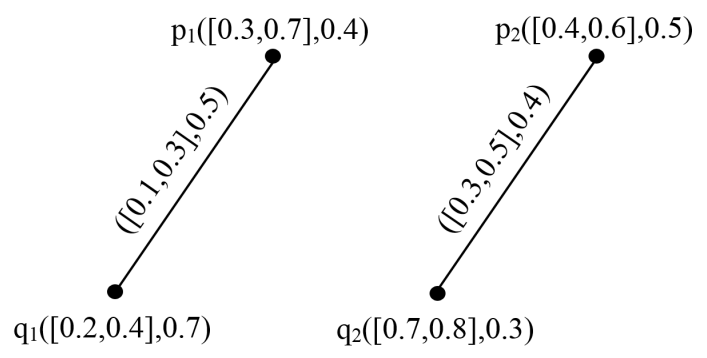

Figure 2. Cubic graphs $M_{1}$ and $M_{2}$

Then, their corresponding Cartesian product $M_{1} \times M_{2}$ is shown in figure 3 .

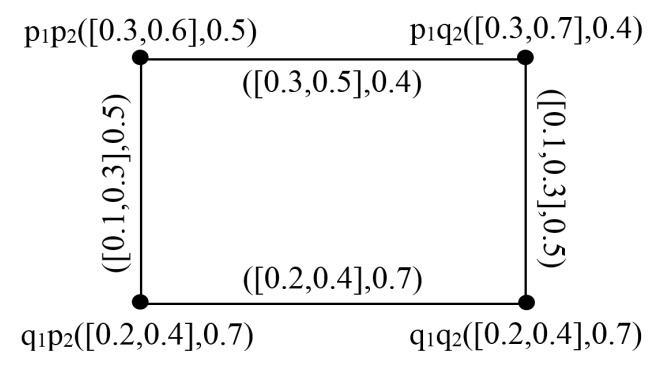

Figure 3. Cubic graph $M_{1} \times M_{2}$

Clearly, $M_{1} \times M_{2}$ is a cubic graph.

Proposition 3.1. The Cartesian product of two cubic graphs is a cubic graph. 
Proof. The conditions for $\alpha_{1} \times \alpha_{2}$ are obvious, therefore, we verify only conditions for $\beta_{1} \times \beta_{2}$.

Let $q \in P_{1}$, and $q_{2} p_{2} \in Q_{2}$. Then

$$
\begin{aligned}
\left(\widetilde{\varpi}_{\beta_{1}} \times \widetilde{\varpi}_{\beta_{2}}\right)\left(\left(q, q_{2}\right)\left(q, p_{2}\right)\right) & =r \min \left\{\widetilde{\varpi}_{\alpha_{1}}(q), \widetilde{\varpi}_{\beta_{2}}\left(q_{2} p_{2}\right)\right\} \\
\preceq & r \min \left\{\widetilde{\varpi}_{\alpha_{1}}(q), r \min \left\{\widetilde{\varpi}_{\alpha_{2}}\left(q_{2}\right), \widetilde{\varpi}_{\alpha_{2}}\left(p_{2}\right)\right\}\right\} \\
& =r \min \left\{r \min \left\{\widetilde{\varpi}_{\alpha_{1}}(q), \widetilde{\varpi}_{\alpha_{2}}\left(q_{2}\right)\right\}, r \min \left\{\widetilde{\varpi}_{\alpha_{1}}(q), \widetilde{\varpi}_{\alpha_{2}}\left(p_{2}\right)\right\}\right\} \\
& =r \min \left\{\left(\widetilde{\varpi}_{\alpha_{1}} \times \widetilde{\varpi}_{\alpha_{2}}\right)\left(q, q_{2}\right),\left(\widetilde{\varpi}_{\alpha_{1}} \times \widetilde{\varpi}_{\alpha_{2}}\right)\left(\left(q, p_{2}\right)\right\}\right. \\
\left(\mu_{\beta_{1}} \times \mu_{\beta_{2}}\right)\left(\left(q, q_{2}\right)\left(q, p_{2}\right)\right) & =\max \left\{\mu_{\alpha_{1}}(q), \mu_{\beta_{2}}\left(q_{2} p_{2}\right)\right\} \\
\leq & \max \left\{\mu_{\alpha_{1}}(q), \max \left\{\mu_{\alpha_{2}}\left(q_{2}\right), \mu_{\alpha_{2}}\left(p_{2}\right)\right\}\right\} \\
& =\max \left\{\max \left\{\mu_{\alpha_{1}}(q), \mu_{\alpha_{2}}\left(q_{2}\right)\right\}, \max \left\{\mu_{\alpha_{1}}(q), \mu_{\alpha_{2}}\left(p_{2}\right)\right\}\right\} \\
& =\max \left\{\left(\mu_{\alpha_{1}} \times \mu_{\alpha_{2}}\right)\left(q, q_{2}\right),\left(\mu_{\alpha_{1}} \times \mu_{\alpha_{2}}\right)\left(\left(q, p_{2}\right)\right\}\right.
\end{aligned}
$$

Similarly, we can prove it for $r \in P_{2}$, and $q_{1} p_{1} \in Q_{1}$.

Definition 3.3. Let $M_{1}=\left\langle\alpha_{1}, \beta_{1}\right\rangle$ and $M_{2}=\left\langle\alpha_{2}, \beta_{2}\right\rangle$ be two cubic graphs of the graphs $\Omega_{1}^{*}$ and $\Omega_{2}^{*}$, respectively. The composition of $M_{1}$ and $M_{2}$ is denoted by $M_{1}\left[M_{2}\right]=\left\langle\alpha_{1} \circ \alpha_{2}, \beta_{1} \circ \beta_{2}\right\rangle$ and is defined as follows:

(i) $\left\{\begin{array}{c}\left(\widetilde{\varpi}_{\alpha_{1}} \circ \widetilde{\varpi}_{\alpha_{2}}\right)\left(p_{1}, p_{2}\right)=r \min \left\{\widetilde{\varpi}_{\alpha_{1}}\left(p_{1}\right), \widetilde{\varpi}_{\alpha_{2}}\left(p_{2}\right)\right\} \\ \left(\mu_{\alpha_{1}} \circ \mu_{\alpha_{2}}\right)\left(p_{1}, p_{2}\right)=\max \left\{\mu_{\alpha_{1}}\left(p_{1}\right), \mu_{\alpha_{2}}\left(p_{2}\right)\right\}\end{array}\right.$

for all $\left(p_{1}, p_{2}\right) \in P=P_{1} \times P_{2}$,

(ii) $\left\{\begin{array}{c}\left(\widetilde{\varpi}_{\beta_{1}} \circ \widetilde{\varpi}_{\beta_{2}}\right)\left(\left(q, q_{2}\right)\left(q, p_{2}\right)\right)=r \min \left\{\widetilde{\varpi}_{\alpha_{1}}(q), \widetilde{\varpi}_{\beta_{2}}\left(q_{2} p_{2}\right)\right\} \\ \left(\mu_{\beta_{1}} \circ \mu_{\beta_{2}}\right)\left(\left(q, q_{2}\right)\left(q, p_{2}\right)\right)=\max \left\{\mu_{\alpha_{1}}(q), \mu_{\beta_{2}}\left(q_{2} p_{2}\right)\right\}\end{array}\right.$

for all $q \in P_{1}$, and $q_{2} p_{2} \in Q_{2}$,

(iii) $\left\{\begin{aligned}\left(\widetilde{\varpi}_{\beta_{1}} \circ \widetilde{\varpi}_{\beta_{2}}\right)\left(\left(q_{1}, r\right)\left(p_{1}, r\right)\right) & =r \min \left\{\widetilde{\varpi}_{\beta_{1}}\left(q_{1} p_{1}\right), \widetilde{\varpi}_{\alpha_{2}}(r)\right\} \\ \left(\mu_{\beta_{1}} \circ \mu_{\beta_{2}}\right)\left(\left(q_{1}, r\right)\left(p_{1}, r\right)\right) & =\max \left\{\mu_{\beta_{1}}\left(q_{1} p_{1}\right), \mu_{\alpha_{2}}(r)\right\}\end{aligned}\right.$

for all $r \in P_{2}$, and $q_{1} p_{1} \in Q_{1}$.

(iv) $\left\{\begin{aligned}\left(\widetilde{\varpi}_{\beta_{1}} \circ \widetilde{\varpi}_{\beta_{2}}\right)\left(\left(q_{1}, q_{2}\right)\left(p_{1}, p_{2}\right)\right) & =r \min \left\{\widetilde{\varpi}_{\alpha_{2}}\left(q_{2}\right), \widetilde{\varpi}_{\alpha_{2}}\left(p_{2}\right), \widetilde{\varpi}_{\beta_{1}}\left(q_{1} p_{1}\right)\right\} \\ \left(\mu_{\beta_{1}} \circ \mu_{\beta_{2}}\right)\left(\left(q_{1}, q_{2}\right)\left(p_{1}, p_{2}\right)\right) & =\max \left\{\mu_{\alpha_{2}}\left(q_{2}\right), \mu_{\alpha_{2}}\left(p_{2}\right), \mu_{\beta_{1}}\left(q_{1} p_{1}\right)\right\}\end{aligned}\right.$

for all $q_{2}, p_{2} \in P_{2}, q_{2} \neq p_{2}$ and $q_{1} p_{1} \in Q_{1}$.

Example 3.4. From Example 3.3, consider two cubic graphs $M_{1}=\left\langle\alpha_{1}, \beta_{1}\right\rangle$ and $M_{2}=\left\langle\alpha_{2}, \beta_{2}\right\rangle$ as shown in figure 2. Then, their corresponding composition $M_{1}\left[M_{2}\right]$ is shown in figure 4 . 


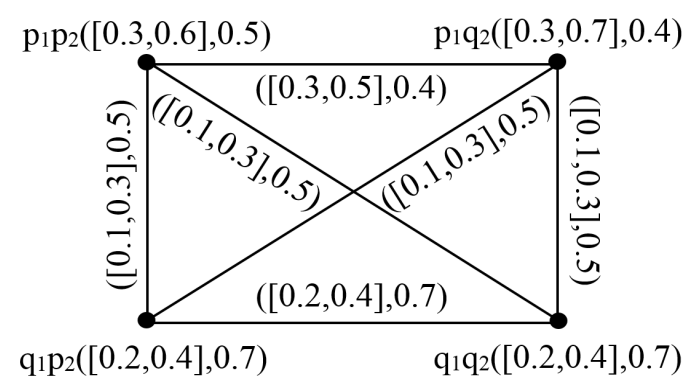

Figure 4. Cubic graph $M_{1}\left[M_{2}\right]$

Clearly, $M_{1}\left[M_{2}\right]$ is a cubic graph.

Proposition 3.2. The composition of two cubic graphs is a cubic graph.

Definition 3.4. Let $M_{1}=\left\langle\alpha_{1}, \beta_{1}\right\rangle$ and $M_{2}=\left\langle\alpha_{2}, \beta_{2}\right\rangle$ be two cubic graphs of the graphs $\Omega_{1}^{*}$ and $\Omega_{2}^{*}$, respectively. The $P$-union of two cubic graphs $M_{1}$ and $M_{2}$ is denoted by $M_{1} \cup_{P} M_{2}=\left\langle\alpha_{1} \cup_{p} \alpha_{2}, \beta_{1} \cup_{p} \beta_{2}\right\rangle$ and is defined as follows:
(i) $\left(\widetilde{\varpi}_{\alpha_{1}} \cup_{p} \widetilde{\varpi}_{\alpha_{2}}\right)(p)= \begin{cases}\widetilde{\varpi}_{\alpha_{1}}(p) & \text { if } p \in P_{1}-P_{2} \\ \widetilde{\varpi}_{\alpha_{2}}(p) & \text { if } p \in P_{2}-P_{1} \\ r \max \left\{\widetilde{\varpi}_{\alpha_{1}}(p), \widetilde{\varpi}_{\alpha_{2}}(p)\right\} & \text { if } p \in P_{1} \cap P_{2}\end{cases}$
(ii) $\left(\mu_{\alpha_{1}} \cup_{p} \mu_{\alpha_{2}}\right)(p)= \begin{cases}\mu_{\alpha_{1}}(p) & \text { if } p \in P_{1}-P_{2} \\ \mu_{\alpha_{2}}(p) & \text { if } p \in P_{2}-P_{1} \\ \max \left\{\mu_{\alpha_{1}}(p), \mu_{\alpha_{2}}(p)\right\} & \text { if } p \in P_{1} \cap P_{2}\end{cases}$
(iii) $\left(\widetilde{\varpi}_{\beta_{1}} \cup_{p} \widetilde{\varpi}_{\beta_{2}}\right)\left(p_{i} p_{j}\right)= \begin{cases}\widetilde{\varpi}_{\beta_{1}}\left(p_{i} p_{j}\right) & \text { if } p_{i} p_{j} \in Q_{1}-Q_{2} \\ \widetilde{\varpi}_{\beta_{2}}\left(p_{i} p_{j}\right) & \text { if } p_{i} p_{j} \in Q_{2}-Q_{1} \\ r \max \left\{\widetilde{\varpi}_{\beta_{1}}\left(p_{i} p_{j}\right), \widetilde{\varpi}_{\beta_{2}}\left(p_{i} p_{j}\right)\right\} & \text { if } p_{i} p_{j} \in Q_{1} \cap Q_{2}\end{cases}$
(iv) $\left(\mu_{\beta_{1}} \cup_{p} \mu_{\beta_{2}}\right)\left(p_{i} p_{j}\right)= \begin{cases}\mu_{\beta_{1}}\left(p_{i} p_{j}\right) & \text { if } p_{i} p_{j} \in Q_{1}-Q_{2} \\ \mu_{\beta_{2}}\left(p_{i} p_{j}\right) & \text { if } p_{i} p_{j} \in Q_{2}-Q_{1} \\ \max \left\{\mu_{\beta_{1}}\left(p_{i} p_{j}\right), \mu_{\beta_{2}}\left(p_{i} p_{j}\right)\right\} & \text { if } p_{i} p_{j} \in Q_{1} \cap Q_{2}\end{cases}$

Definition 3.5. Let $M_{1}=\left\langle\alpha_{1}, \beta_{1}\right\rangle$ and $M_{2}=\left\langle\alpha_{2}, \beta_{2}\right\rangle$ be two cubic graphs of the graphs $\Omega_{1}^{*}$ and $\Omega_{2}^{*}$, respectively. The $R$-union of two cubic graphs $M_{1}$ and $M_{2}$ is denoted by $M_{1} \cup_{R} M_{2}=\left\langle\alpha_{1} \cup_{R} \alpha_{2}, \beta_{1} \cup_{R} \beta_{2}\right\rangle$ and is defined as follows:
(i) $\left(\widetilde{\varpi}_{\alpha_{1}} \cup_{R} \widetilde{\varpi}_{\alpha_{2}}\right)(p)= \begin{cases}\widetilde{\varpi}_{\alpha_{1}}(p) & \text { if } p \in P_{1}-P_{2} \\ \widetilde{\varpi}_{\alpha_{2}}(p) & \text { if } p \in P_{2}-P_{1} \\ r \max \left\{\widetilde{\varpi}_{\alpha_{1}}(p), \widetilde{\varpi}_{\alpha_{2}}(p)\right\} & \text { if } p \in P_{1} \cap P_{2}\end{cases}$ 
(ii) $\left(\mu_{\alpha_{1}} \cup_{R} \mu_{\alpha_{2}}\right)(p)= \begin{cases}\mu_{\alpha_{1}}(p) & \text { if } p \in P_{1}-P_{2} \\ \mu_{\alpha_{2}}(p) & \text { if } p \in P_{2}-P_{1} \\ \min \left\{\mu_{\alpha_{1}}(p), \mu_{\alpha_{2}}(p)\right\} & \text { if } p \in P_{1} \cap P_{2}\end{cases}$

(iii) $\left(\widetilde{\varpi}_{\beta_{1}} \cup_{R} \widetilde{\varpi}_{\beta_{2}}\right)\left(p_{i} p_{j}\right)= \begin{cases}\widetilde{\varpi}_{\beta_{1}}\left(p_{i} p_{j}\right) & \text { if } p_{i} p_{j} \in Q_{1}-Q_{2} \\ \widetilde{\varpi}_{\beta_{2}}\left(p_{i} p_{j}\right) & \text { if } p_{i} p_{j} \in Q_{2}-Q_{1} \\ r \max \left\{\widetilde{\varpi}_{\beta_{1}}\left(p_{i} p_{j}\right), \widetilde{\varpi}_{\beta_{2}}\left(p_{i} p_{j}\right)\right\} & \text { if } p_{i} p_{j} \in Q_{1} \cap Q_{2}\end{cases}$

(iv) $\left(\mu_{\beta_{1}} \cup_{R} \mu_{\beta_{2}}\right)\left(p_{i} p_{j}\right)= \begin{cases}\mu_{\beta_{1}}\left(p_{i} p_{j}\right) & \text { if } p_{i} p_{j} \in Q_{1}-Q_{2} \\ \mu_{\beta_{2}}\left(p_{i} p_{j}\right) & \text { if } p_{i} p_{j} \in Q_{2}-Q_{1} \\ \min \left\{\mu_{\beta_{1}}\left(p_{i} p_{j}\right), \mu_{\beta_{2}}\left(p_{i} p_{j}\right)\right\} & \text { if } p_{i} p_{j} \in Q_{1} \cap Q_{2}\end{cases}$

Example 3.5. Consider two cubic graphs $M_{1}=\left\langle\alpha_{1}, \beta_{1}\right\rangle$ and $M_{2}=\left\langle\alpha_{2}, \beta_{2}\right\rangle$ as shown in figure 5 .
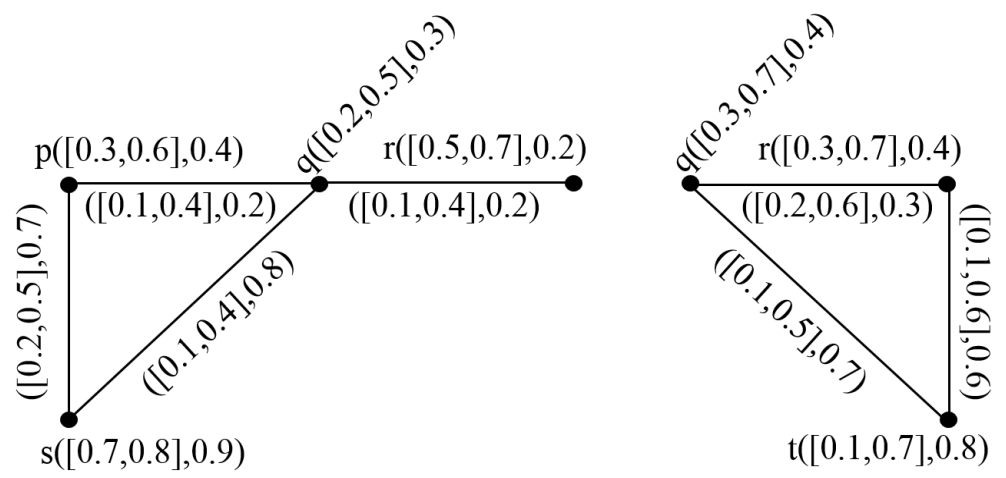

Figure 5. Cubic graphs $M_{1}$ and $M_{2}$

Then, their corresponding $P$-union $M_{1} \cup_{P} M_{2}$ is shown in figure 6 .

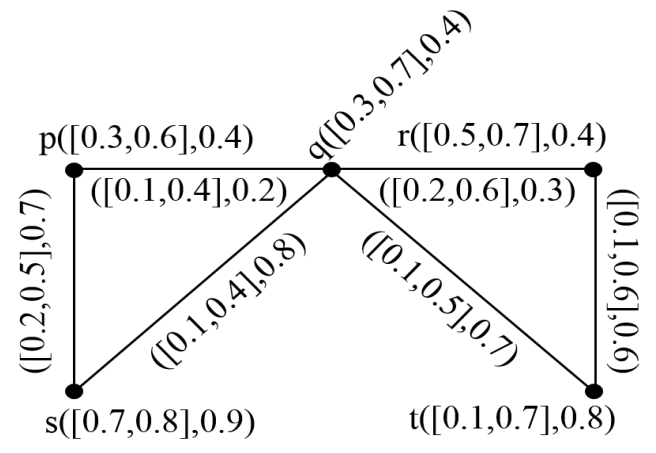

Figure 6. Cubic graph $M_{1} \cup_{P} M_{2}$

Also, their corresponding $R$-union $M_{1} \cup_{R} M_{2}$ is shown in figure 7 . 


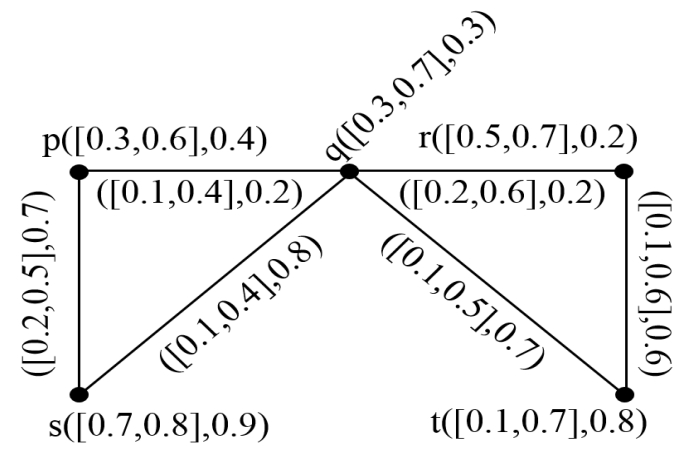

Figure 7. Cubic graph $M_{1} \cup_{R} M_{2}$

Clearly, $M_{1} \cup_{P} M_{2}$ and $M_{1} \cup_{R} M_{2}$ are cubic graphs.

Proposition 3.3. The $P$-union and $R$-union of two cubic graphs is a cubic graph.

Proof. Since all the conditions for $\alpha_{1} \cup_{p} \alpha_{2}$ are automatically satisfied therefore, we verify only conditions for $\beta_{1} \cup_{p} \beta_{2}$. In the case, when $q p \in Q_{1} \cap Q_{2}$. Then

$$
\begin{aligned}
\left(\widetilde{\varpi}_{\beta_{1}} \cup_{p} \widetilde{\varpi}_{\beta_{2}}\right)(q p) & =r \max \left\{\widetilde{\varpi}_{\beta_{1}}(q p), \widetilde{\varpi}_{\beta_{2}}(q p)\right\} \\
\preceq & r \max \left\{r \min \left\{\widetilde{\varpi}_{\alpha_{1}}(q), \widetilde{\varpi}_{\alpha_{1}}(p)\right\}, r \min \left\{\widetilde{\varpi}_{\alpha_{2}}(q), \widetilde{\varpi}_{\alpha_{2}}(p)\right\}\right\} \\
= & r \min \left\{r \max \left\{\widetilde{\varpi}_{\alpha_{1}}(q), \widetilde{\varpi}_{\alpha_{2}}(q)\right\}, r \max \left\{\widetilde{\varpi}_{\alpha_{1}}(p), \widetilde{\varpi}_{\alpha_{2}}(p)\right\}\right\} \\
= & r \min \left\{\left(\widetilde{\varpi}_{\alpha_{1}} \cup_{p} \widetilde{\varpi}_{\alpha_{2}}\right)(q),\left(\widetilde{\varpi}_{\alpha_{1}} \cup_{p} \widetilde{\varpi}_{\alpha_{2}}\right)(p)\right\} . \\
\left(\mu_{\beta_{1}} \cup_{p} \mu_{\beta_{2}}\right)(q p) & =\max \left\{\mu_{\beta_{1}}(q p), \mu_{\beta_{2}}(q p)\right\} \\
& \leq \max \left\{\max \left\{\mu_{\alpha_{1}}(q), \mu_{\alpha_{1}}(p)\right\}, \max \left\{\mu_{\alpha_{2}}(q), \mu_{\alpha_{2}}(p)\right\}\right\} \\
& =\max \left\{\max \left\{\mu_{\alpha_{1}}(q), \mu_{\alpha_{2}}(q)\right\}, \max \left\{\mu_{\alpha_{1}}(p), \mu_{\alpha_{2}}(p)\right\}\right\} \\
& =\max \left\{\left(\mu_{\alpha_{1}} \cup_{p} \mu_{\alpha_{2}}\right)(q),\left(\mu_{\alpha_{1}} \cup_{p} \mu_{\alpha_{2}}\right)(p)\right\} .
\end{aligned}
$$

If $q p \in Q_{1}$ and $q p \notin Q_{2}$, then

$$
\begin{aligned}
\left(\widetilde{\varpi}_{\beta_{1}} \cup_{p} \widetilde{\varpi}_{\beta_{2}}\right)(q p) & \preceq r \min \left\{\left(\widetilde{\varpi}_{\alpha_{1}} \cup_{p} \widetilde{\varpi}_{\alpha_{2}}\right)(q),\left(\widetilde{\varpi}_{\alpha_{1}} \cup_{p} \widetilde{\varpi}_{\alpha_{2}}\right)(p)\right\} \\
\left(\mu_{\beta_{1}} \cup_{p} \mu_{\beta_{2}}\right)(q p) & \leq \max \left\{\left(\mu_{\alpha_{1}} \cup_{p} \mu_{\alpha_{2}}\right)(q),\left(\mu_{\alpha_{1}} \cup_{p} \mu_{\alpha_{2}}\right)(p)\right\} .
\end{aligned}
$$

If $q p \in Q_{2}$ and $q p \notin Q_{1}$, then

$$
\begin{aligned}
\left(\widetilde{\varpi}_{\beta_{1}} \cup_{p} \widetilde{\varpi}_{\beta_{2}}\right)(q p) & \preceq r \min \left\{\left(\widetilde{\varpi}_{\alpha_{1}} \cup_{p} \widetilde{\varpi}_{\alpha_{2}}\right)(q),\left(\widetilde{\varpi}_{\alpha_{1}} \cup_{p} \widetilde{\varpi}_{\alpha_{2}}\right)(p)\right\} \\
\left(\mu_{\beta_{1}} \cup_{p} \mu_{\beta_{2}}\right)(q p) & \leq \max \left\{\left(\mu_{\alpha_{1}} \cup_{p} \mu_{\alpha_{2}}\right)(q),\left(\mu_{\alpha_{1}} \cup_{p} \mu_{\alpha_{2}}\right)(p)\right\} .
\end{aligned}
$$


Hence the $P$-union of two cubic graphs is a cubic graph. The case for $R$-union of two cubic graphs can be seen in a similar way.

Definition 3.6. Let $M_{1}=\left\langle\alpha_{1}, \beta_{1}\right\rangle$ and $M_{2}=\left\langle\alpha_{2}, \beta_{2}\right\rangle$ be two cubic graphs of the graphs $\Omega_{1}^{*}$ and $\Omega_{2}^{*}$, respectively. The $P$-join of two cubic graphs $M_{1}$ and $M_{2}$ is denoted by $M_{1}+_{P} M_{2}=\left\langle\alpha_{1}+_{P} \alpha_{2}, \beta_{1}+_{P} \beta_{2}\right\rangle$ and is defined as follows:

(i) $\left\{\begin{aligned}\left(\widetilde{\varpi}_{\alpha_{1}}+{ }_{P} \widetilde{\varpi}_{\alpha_{2}}\right)(p) & =\left(\widetilde{\varpi}_{\alpha_{1}} \cup_{P} \widetilde{\varpi}_{\alpha_{2}}\right)(p) \\ \left(\mu_{\alpha_{1}}+{ }_{P} \mu_{\alpha_{2}}\right)(p) & =\left(\mu_{\alpha_{1}} \cup_{P} \mu_{\alpha_{2}}\right)(p)\end{aligned}\right.$

for $p \in P_{1} \cup P_{2}$,

(ii) $\left\{\begin{aligned}\left(\widetilde{\varpi}_{\beta_{1}}+P \widetilde{\varpi}_{\beta_{2}}\right)(q p) & =\left(\widetilde{\varpi}_{\beta_{1}} \cup_{P} \widetilde{\varpi}_{\beta_{2}}\right)(q p) \\ \left(\mu_{\beta_{1}}+{ }_{P} \mu_{\beta_{2}}\right)(q p) & =\left(\mu_{\beta_{1}} \cup_{P} \mu_{\beta_{2}}\right)(q p)\end{aligned}\right.$

for $q p \in Q_{1} \cap Q_{2}$,

(iii) $\left\{\begin{array}{c}\left(\widetilde{\varpi}_{\beta_{1}}+{ }_{P} \widetilde{\varpi}_{\beta_{2}}\right)(q p)=r \min \left\{\widetilde{\varpi}_{\alpha_{1}}(q), \widetilde{\varpi}_{\alpha_{2}}(p)\right\} \\ \left(\mu_{\beta_{1}}+{ }_{P} \mu_{\beta_{2}}\right)(q p)=\min \left\{\mu_{\alpha_{1}}(q), \mu_{\alpha_{2}}(p)\right\}\end{array}\right.$

for $q p \in Q^{*}$, where $Q^{*}$ is the set of all edges joining the vertices of $P_{1}$ and $P_{2}$.

Definition 3.7. Let $M_{1}=\left\langle\alpha_{1}, \beta_{1}\right\rangle$ and $M_{2}=\left\langle\alpha_{2}, \beta_{2}\right\rangle$ be two cubic graphs of the graphs $\Omega_{1}^{*}$ and $\Omega_{2}^{*}$, respectively. The $R$-join of two cubic graphs $M_{1}$ and $M_{2}$ is denoted by $M_{1}+_{R} M_{2}=\left\langle\alpha_{1}+_{R} \alpha_{2}, \beta_{1}+_{R} \beta_{2}\right\rangle$ and is defined as follows:

(i) $\left\{\begin{aligned}\left(\widetilde{\varpi}_{\alpha_{1}}+R \widetilde{\varpi}_{\alpha_{2}}\right)(p) & =\left(\widetilde{\varpi}_{\alpha_{1}} \cup_{R} \widetilde{\varpi}_{\alpha_{2}}\right)(p) \\ \left(\mu_{\alpha_{1}}+R\right. & \left.\mu_{\alpha_{2}}\right)(p)=\left(\mu_{\alpha_{1}} \cup_{R} \mu_{\alpha_{2}}\right)(p)\end{aligned}\right.$

for $p \in P_{1} \cup P_{2}$,

(ii) $\left\{\begin{aligned}\left(\widetilde{\varpi}_{\beta_{1}}+{ }_{R} \widetilde{\varpi}_{\beta_{2}}\right)(q p) & =\left(\widetilde{\varpi}_{\beta_{1}} \cup_{R} \widetilde{\varpi}_{\beta_{2}}\right)(q p) \\ \left(\mu_{\beta_{1}}+{ }_{R} \mu_{\beta_{2}}\right)(q p) & =\left(\mu_{\beta_{1}} \cup_{R} \mu_{\beta_{2}}\right)(q p)\end{aligned}\right.$

for $q p \in Q_{1} \cap Q_{2}$,

(iii) $\left\{\begin{array}{c}\left(\widetilde{\varpi}_{\beta_{1}}+{ }_{R} \widetilde{\varpi}_{\beta_{2}}\right)(q p)=r \min \left\{\widetilde{\varpi}_{\alpha_{1}}(q), \widetilde{\varpi}_{\alpha_{2}}(p)\right\} \\ \left(\mu_{\beta_{1}}+R \mu_{\beta_{2}}\right)(q p)=\max \left\{\mu_{\alpha_{1}}(q), \mu_{\alpha_{2}}(p)\right\}\end{array}\right.$

for $q p \in Q^{*}$, where $Q^{*}$ is the set of all edges joining the vertices of $P_{1}$ and $P_{2}$.

Example 3.6. Consider two cubic graphs $M_{1}=\left\langle\alpha_{1}, \beta_{1}\right\rangle$ and $M_{2}=\left\langle\alpha_{2}, \beta_{2}\right\rangle$ as shown in figure 8 .

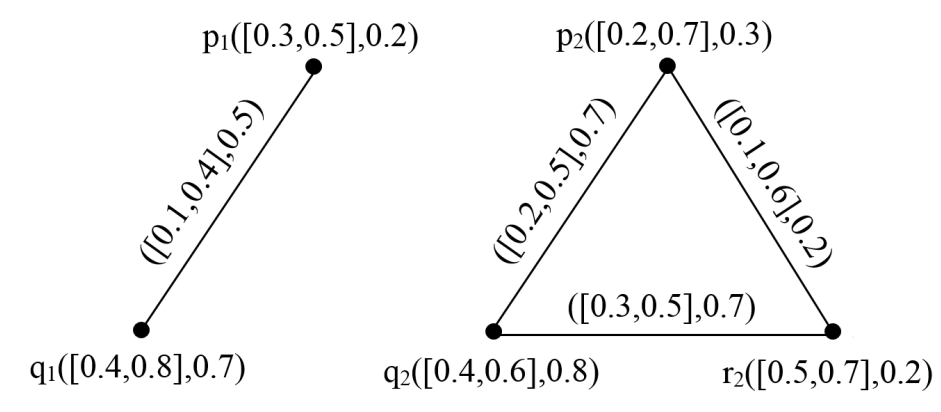

Figure 8. Cubic graphs $M_{1}$ and $M_{2}$ 
Then, their corresponding $P$-join $M_{1}+{ }_{P} M_{2}$ is shown in figure 9 .

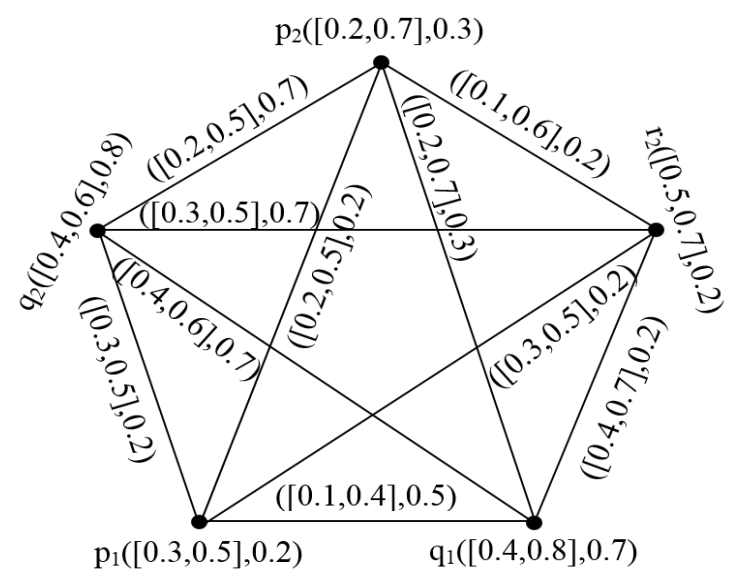

Figure 9. Cubic graph $M_{1}+{ }_{P} M_{2}$

Also, their corresponding $R$-join $M_{1}+{ }_{R} M_{2}$ is shown in figure 10 .

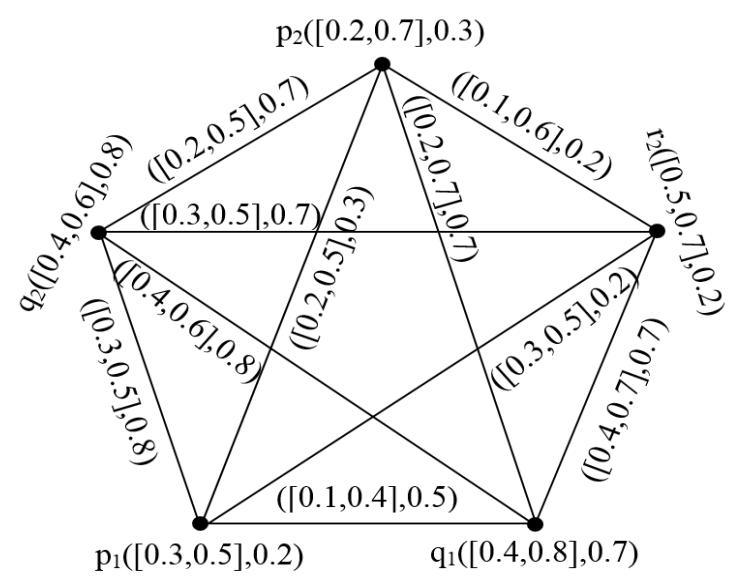

Figure 10. Cubic graph $M_{1}+{ }_{R} M_{2}$

Clearly, $M_{1}+_{P} M_{2}$ and $M_{1}+{ }_{R} M_{2}$ are cubic graphs.

Proposition 3.4. The $P$-join and $R$-join of two cubic graphs is a cubic graph.

4. INTERNAL AND EXTERNAL CUBIC GRAPHS

Here in this section we discuss some results related with internal and external cubic graphs.

Definition 4.1. A cubic graph $M=\langle\alpha, \beta\rangle$ is said to be an

(i) internal cubic graph (IC-graph) if

$$
\mu_{\alpha}\left(p_{i}\right) \in\left[\varpi_{\alpha}^{-}\left(p_{i}\right), \varpi_{\alpha}^{+}\left(p_{i}\right)\right] \text { and } \mu_{\beta}\left(e_{i}\right) \in\left[\varpi_{\beta}^{-}\left(e_{i}\right), \varpi_{\beta}^{+}\left(e_{i}\right)\right]
$$


for each $p_{i} \in P$ and $e_{i} \in Q$.

(ii) external cubic graph (EC-graph) if

$$
\mu_{\alpha}\left(p_{i}\right) \notin\left(\varpi_{\alpha}^{-}\left(p_{i}\right), \varpi_{\alpha}^{+}\left(p_{i}\right)\right) \text { and } \mu_{\beta}\left(e_{i}\right) \notin\left(\varpi_{\beta}^{-}\left(e_{i}\right), \varpi_{\beta}^{+}\left(e_{i}\right)\right)
$$

for each $p_{i} \in P$ and $e_{i} \in Q$.

Example 4.1. The cubic graphs $M_{1}=\left\langle\alpha_{1}, \beta_{1}\right\rangle$ and $M_{2}=\left\langle\alpha_{2}, \beta_{2}\right\rangle$ are internal and external cubic graphs, respectively, as shown in figure 11.

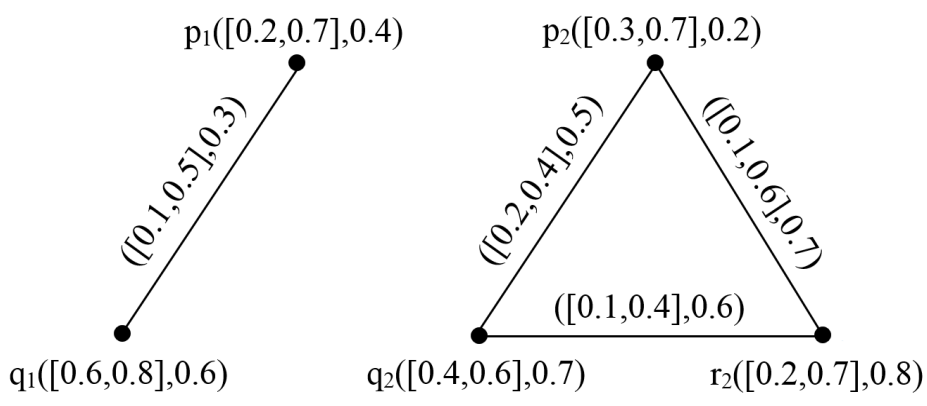

Figure 11. IC-graph $M_{1}$ and EC-graph $M_{2}$

Theorem 4.1. Let $\left\{M_{i}=\left\langle\alpha_{i}, \beta_{i}\right\rangle \mid i \in I\right\}$ be a family of IC-graphs. Then $\cup_{i \in I} M_{i}$ is an IC-graph.

Proof. Since $M_{i}$ is an IC-graph, we have $\varpi_{\alpha}^{-}(p) \leq \mu_{\alpha}(p) \leq \varpi_{\alpha}^{+}(p)$ and $\varpi_{\beta}^{-}(e) \leq \mu_{\beta}(e) \leq \varpi_{\beta}^{+}(e)$ for $i \in I$. This implies that

$$
\left(\cup_{i \in I} \varpi_{\alpha}^{-}\right)(p) \leq\left(\bigvee_{i \in I} \mu_{\alpha}\right)(p) \leq\left(\cup_{i \in I} \varpi_{\alpha}^{+}\right)(p)
$$

and

$$
\left(\cup_{i \in I} \varpi_{\beta}^{-}\right)(e) \leq\left(\bigvee_{i \in I} \mu_{\beta}\right)(e) \leq\left(\cup_{i \in I} \varpi_{\beta}^{+}\right)(e)
$$

Hence $\cup_{i \in I} M_{i}$ is an IC-graph.

The following example shows that the $R$-union of IC-graphs need not be an IC-graph (EC-graph).

Example 4.2. Consider two IC-graphs $M_{1}=\left\langle\alpha_{1}, \beta_{1}\right\rangle$ and $M_{2}=\left\langle\alpha_{2}, \beta_{2}\right\rangle$ as shown in figure 12 . 


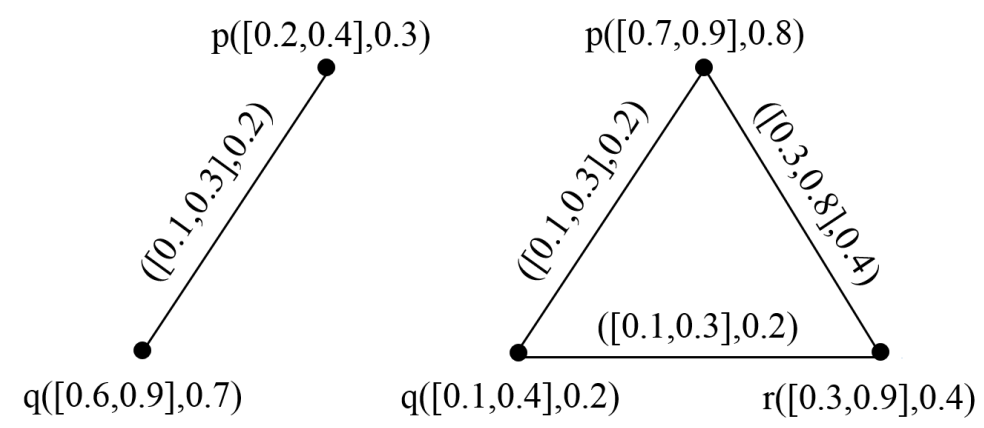

FiguRE 12. IC-graphs $M_{1}$ and $M_{2}$

Then, their corresponding $R$-union $M_{1} \cup_{R} M_{2}$ is shown in figure 13 .

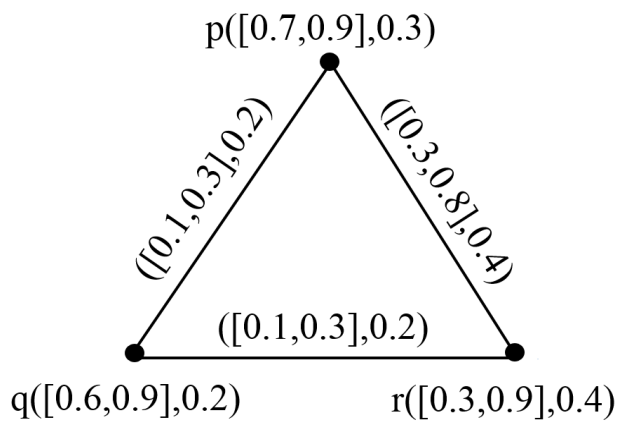

Figure 13. $R$-union of IC-graphs $M_{1}$ and $M_{2}$

It is easy to see that the cubic graph $M_{1} \cup_{R} M_{2}$ is neither IC-graph nor EC-graph.

We provide a condition for the $R$-union of two IC-graphs to be an IC-graph.

Theorem 4.2. Let $M_{1}=\left\langle\alpha_{1}, \beta_{1}\right\rangle$ and $M_{2}=\left\langle\alpha_{2}, \beta_{2}\right\rangle$ be IC-graphs such that

$$
\max \left\{\varpi_{\alpha_{1}}^{-}(p), \varpi_{\alpha_{2}}^{-}(p)\right\} \leq \min \left\{\mu_{\alpha_{1}}(p), \mu_{\alpha_{2}}(p)\right\}
$$

and

$$
\max \left\{\varpi_{\beta_{1}}^{-}(e), \varpi_{\beta_{2}}^{-}(e)\right\} \leq \min \left\{\mu_{\beta_{1}}(e), \mu_{\beta_{2}}(e)\right\}
$$

for all $p \in P$ and $e \in Q$. Then the $R$-union of two IC-graphs $M_{1}$ and $M_{2}$ is an IC-graph.

Proof. Let $M_{1}=\left\langle\alpha_{1}, \beta_{1}\right\rangle$ and $M_{2}=\left\langle\alpha_{2}, \beta_{2}\right\rangle$ be two IC-graphs which satisfy the conditions

$$
\max \left\{\varpi_{\alpha_{1}}^{-}(p), \varpi_{\alpha_{2}}^{-}(p)\right\} \leq \min \left\{\mu_{\alpha_{1}}(p), \mu_{\alpha_{2}}(p)\right\}
$$

and

$$
\max \left\{\varpi_{\beta_{1}}^{-}(e), \varpi_{\beta_{2}}^{-}(e)\right\} \leq \min \left\{\mu_{\beta_{1}}(e), \mu_{\beta_{2}}(e)\right\}
$$


for all $p \in P$ and $e \in Q$. Since $\mu_{\alpha_{1}}(p) \in\left[\varpi_{\alpha_{1}}^{-}(p), \varpi_{\alpha_{1}}^{+}(p)\right], \mu_{\beta_{1}}(e) \in\left[\varpi_{\beta_{1}}^{-}(e), \varpi_{\beta_{1}}^{+}(e)\right]$ and $\mu_{\alpha_{2}}(p) \in$ $\left[\varpi_{\alpha_{2}}^{-}(p), \varpi_{\alpha_{2}}^{+}(p)\right], \mu_{\beta_{2}}(e) \in\left[\varpi_{\beta_{2}}^{-}(e), \varpi_{\beta_{2}}^{+}(e)\right]$. This implies that

$$
\min \left\{\mu_{\alpha_{1}}(p), \mu_{\alpha_{2}}(p)\right\} \leq\left(\varpi_{\alpha_{1}}^{+} \cup \varpi_{\alpha_{2}}^{+}\right)(p) \text { and } \min \left\{\mu_{\beta_{1}}(e), \mu_{\beta_{2}}(e)\right\} \leq\left(\varpi_{\beta_{1}}^{+} \cup \varpi_{\beta_{2}}^{+}\right)(e)
$$

Thus from the given condition we get

$$
\left(\varpi_{\alpha_{1}}^{-} \cup \varpi_{\alpha_{2}}^{-}\right)(p)=\max \left\{\varpi_{\alpha_{1}}^{-}(p), \varpi_{\alpha_{2}}^{-}(p)\right\} \leq \min \left\{\mu_{\alpha_{1}}(p), \mu_{\alpha_{2}}(p)\right\} \leq\left(\varpi_{\alpha_{1}}^{+} \cup \varpi_{\alpha_{2}}^{+}\right)(p),
$$

and

$$
\left(\varpi_{\beta_{1}}^{-} \cup \varpi_{\beta_{2}}^{-}\right)(e)=\max \left\{\varpi_{\beta_{1}}^{-}(e), \varpi_{\beta_{2}}^{-}(e)\right\} \leq \min \left\{\mu_{\beta_{1}}(e), \mu_{\beta_{2}}(e)\right\} \leq\left(\varpi_{\beta_{1}}^{+} \cup \varpi_{\beta_{2}}^{+}\right)(e) .
$$

This shows that $M_{1} \cup_{R} M_{2}$ is an IC-graph.

The following example shows that the $P$-union and $R$-union of EC-graphs need not be an EC-graph (IC-graph).

Example 4.3. Consider two EC-graphs $M_{1}=\left\langle\alpha_{1}, \beta_{1}\right\rangle$ and $M_{2}=\left\langle\alpha_{2}, \beta_{2}\right\rangle$ as shown in figure 14 .

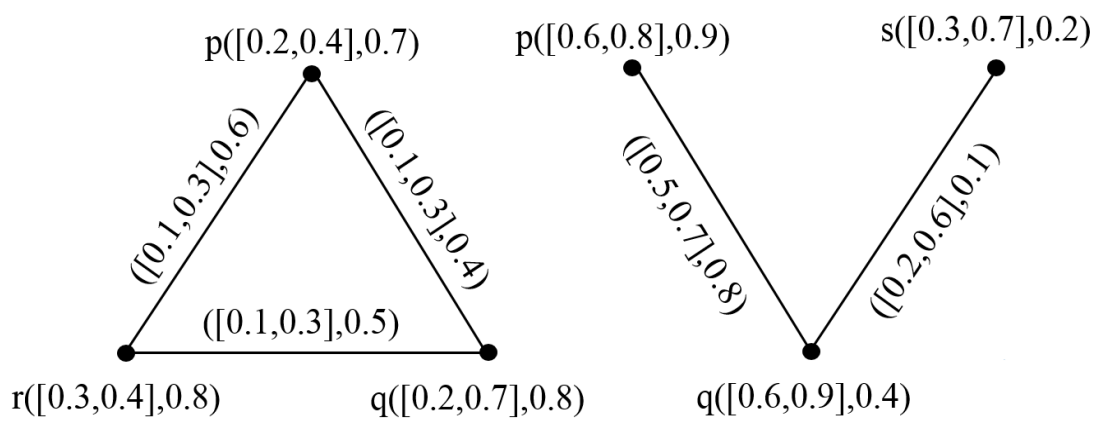

FIgURE 14. EC-graphs $M_{1}$ and $M_{2}$

Then, their corresponding $P$-union $M_{1} \cup_{P} M_{2}$ is shown in figure 15 .

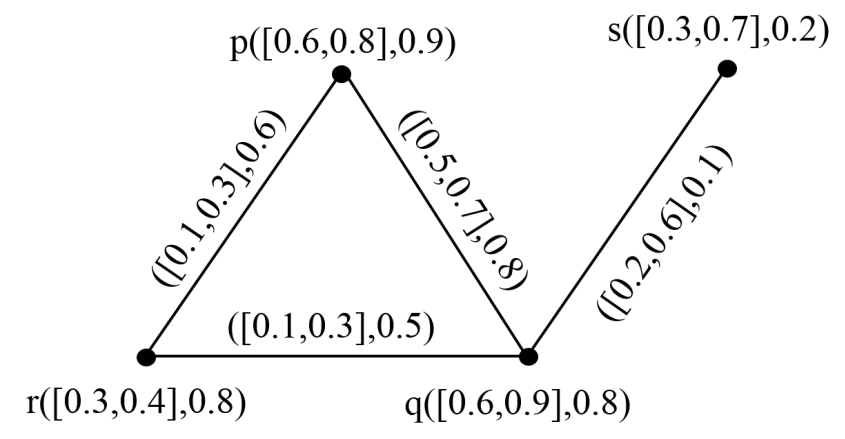

Figure 15. $P$-union of EC-graphs $M_{1}$ and $M_{2}$

Also, the corresponding $R$-union $M_{1} \cup_{R} M_{2}$ is shown in figure 16 . 


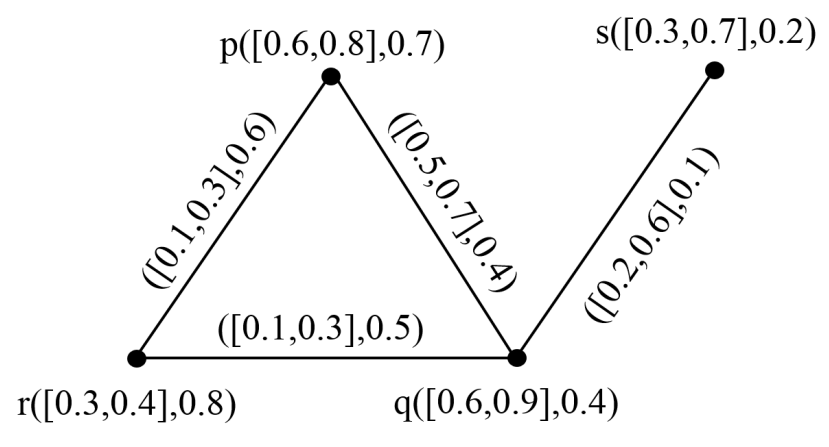

FIGURE 16. $R$-union of EC-graphs $M_{1}$ and $M_{2}$

It is easy to see that the cubic graph $M_{1} \cup_{P} M_{2}$ and $M_{1} \cup_{R} M_{2}$ are neither EC-graph nor IC-graph.

We provide a condition for the $P$-union of two EC-graphs to be an EC-graph.

Theorem 4.3. Let $M_{1}=\left\langle\alpha_{1}, \beta_{1}\right\rangle$ and $M_{2}=\left\langle\alpha_{2}, \beta_{2}\right\rangle$ be two EC-graphs such that

$$
\begin{aligned}
\min \left\{\begin{array}{c}
\max \left\{\varpi_{\alpha_{1}}^{+}(p), \varpi_{\alpha_{2}}^{-}(p)\right\}, \\
\max \left\{\varpi_{\alpha_{1}}^{-}(p), \varpi_{\alpha_{2}}^{+}(p)\right\}
\end{array}\right\} & >\max \left\{\mu_{\alpha_{1}}(p), \mu_{\alpha_{2}}(p)\right\} \\
& \geq \max \left\{\begin{array}{c}
\min \left\{\varpi_{\alpha_{1}}^{+}(p), \varpi_{\alpha_{2}}^{-}(p)\right\}, \\
\min \left\{\varpi_{\alpha_{1}}^{-}(p), \varpi_{\alpha_{2}}^{+}(p)\right\}
\end{array}\right\}
\end{aligned}
$$

and

$$
\begin{aligned}
\min \left\{\begin{array}{c}
\max \left\{\varpi_{\beta_{1}}^{+}(e), \varpi_{\beta_{2}}^{-}(e)\right\}, \\
\max \left\{\varpi_{\beta_{1}}^{+}(e), \varpi_{\beta_{2}}^{-}(e)\right\}
\end{array}\right\} & >\max \left\{\mu_{\beta_{1}}(e), \mu_{\beta_{2}}(e)\right\} \\
& \geq \max \left\{\begin{array}{c}
\min \left\{\varpi_{\beta_{1}}^{+}(e), \varpi_{\beta_{2}}^{-}(e)\right\}, \\
\min \left\{\varpi_{\beta_{1}}^{+}(e), \varpi_{\beta_{2}}^{-}(e)\right\}
\end{array}\right\}
\end{aligned}
$$

for all $p \in P$ and $e \in Q$. Then the $P$-union of two EC-graphs is an EC-graph.

We provide a condition for the $R$-union of two EC-graphs to be an EC-graph.

Theorem 4.4. Let $M_{1}=\left\langle\alpha_{1}, \beta_{1}\right\rangle$ and $M_{2}=\left\langle\alpha_{2}, \beta_{2}\right\rangle$ be two EC-graphs such that

$$
\begin{aligned}
\min \left\{\begin{array}{c}
\max \left\{\varpi_{\alpha_{1}}^{+}(p), \varpi_{\alpha_{2}}^{-}(p)\right\}, \\
\max \left\{\varpi_{\alpha_{1}}^{-}(p), \varpi_{\alpha_{2}}^{+}(p)\right\}
\end{array}\right\} & >\min \left\{\mu_{\alpha_{1}}(p), \mu_{\alpha_{2}}(p)\right\} \\
& \geq \max \left\{\begin{array}{c}
\min \left\{\varpi_{\alpha_{1}}^{+}(p), \varpi_{\alpha_{2}}^{-}(p)\right\}, \\
\min \left\{\varpi_{\alpha_{1}}^{-}(p), \varpi_{\alpha_{2}}^{+}(p)\right\}
\end{array}\right\}
\end{aligned}
$$


and

$$
\begin{aligned}
\min \left\{\begin{array}{c}
\max \left\{\varpi_{\beta_{1}}^{+}(e), \varpi_{\beta_{2}}^{-}(e)\right\}, \\
\max \left\{\varpi_{\beta_{1}}^{+}(e), \varpi_{\beta_{2}}^{-}(e)\right\}
\end{array}\right\} & >\min \left\{\mu_{\beta_{1}}(e), \mu_{\beta_{2}}(e)\right\} \\
& \geq \max \left\{\begin{array}{c}
\min \left\{\varpi_{\beta_{1}}^{+}(e), \varpi_{\beta_{2}}^{-}(e)\right\}, \\
\min \left\{\varpi_{\beta_{1}}^{+}(e), \varpi_{\beta_{2}}^{-}(e)\right\}
\end{array}\right\}
\end{aligned}
$$

for all $p \in P$ and $e \in Q$. Then the $R$-union of two EC-graphs is an EC-graph.

Theorem 4.5. Let $M=\langle\alpha, \beta\rangle$ be a cubic graph which is not an EC-graph. Then there exist $p_{i} \in P$ and $e_{i} \in Q$ such that

$$
\mu_{\alpha}\left(p_{i}\right) \in\left(\varpi_{\alpha}^{-}\left(p_{i}\right), \varpi_{\alpha}^{+}\left(p_{i}\right)\right) \text { and } \mu_{\beta}\left(e_{i}\right) \in\left(\varpi_{\beta}^{-}\left(e_{i}\right), \varpi_{\beta}^{+}\left(e_{i}\right)\right)
$$

Proof. Straightforward.

Theorem 4.6. Let $M=\langle\alpha, \beta\rangle$ be a cubic graph of $\Omega^{*}$. If $M=\langle\alpha, \beta\rangle$ is both an IC-graph and an EC-graph, then

$$
\mu_{\alpha}\left(p_{i}\right) \in U\left(\widetilde{\varpi}_{\alpha}\right) \cup L\left(\widetilde{\varpi}_{\alpha}\right)
$$

and

$$
\mu_{\beta}\left(e_{i}\right) \in U\left(\widetilde{\varpi}_{\beta}\right) \cup L\left(\widetilde{\varpi}_{\beta}\right)
$$

for all $p_{i} \in P$ and $e_{i} \in Q \subseteq P \times P$. Where

$$
U\left(\widetilde{\varpi}_{\alpha}\right)=\left\{\varpi_{\alpha}^{+}\left(p_{i}\right) \mid p_{i} \in P\right\}, \quad L\left(\widetilde{\varpi}_{\alpha}\right)=\left\{\varpi_{\alpha}^{-}\left(p_{i}\right) \mid p_{i} \in P\right\}
$$

and

$$
U\left(\widetilde{\varpi}_{\beta}\right)=\left\{\varpi_{\beta}^{+}\left(e_{i}\right) \mid e_{i} \in Q\right\}, L\left(\widetilde{\varpi}_{\beta}\right)=\left\{\varpi_{\beta}^{-}\left(e_{i}\right) \mid e_{i} \in Q\right\}
$$

Proof. Assume that $M=\langle\alpha, \beta\rangle$ is both an IC-graph and an EC-graph. Then by definition we have

$$
\mu_{\alpha}\left(p_{i}\right) \in\left[\varpi_{\alpha}^{-}\left(p_{i}\right), \varpi_{\alpha}^{+}\left(p_{i}\right)\right], \mu_{\beta}\left(e_{i}\right) \in\left[\varpi_{\beta}^{-}\left(e_{i}\right), \varpi_{\beta}^{+}\left(e_{i}\right)\right]
$$

and

$$
\mu_{\alpha}\left(p_{i}\right) \notin\left(\varpi_{\alpha}^{-}\left(p_{i}\right), \varpi_{\alpha}^{+}\left(p_{i}\right)\right), \mu_{\beta}\left(e_{i}\right) \notin\left(\varpi_{\beta}^{-}\left(e_{i}\right), \varpi_{\beta}^{+}\left(e_{i}\right)\right) .
$$

Thus $\mu_{\alpha}\left(p_{i}\right)=\varpi_{\alpha}^{-}\left(p_{i}\right)$ or $\mu_{\alpha}\left(p_{i}\right)=\varpi_{\alpha}^{+}\left(p_{i}\right)$ and $\mu_{\beta}\left(e_{i}\right)=\varpi_{\beta}^{-}\left(e_{i}\right)$ or $\mu_{\beta}\left(e_{i}\right)=\varpi_{\beta}^{+}\left(e_{i}\right)$. Hence $\mu_{\alpha}\left(p_{i}\right) \in$ $U\left(\widetilde{\varpi}_{\alpha}\right) \cup L\left(\widetilde{\varpi}_{\alpha}\right)$ and $\mu_{\beta}\left(e_{i}\right) \in U\left(\widetilde{\varpi}_{\beta}\right) \cup L\left(\widetilde{\varpi}_{\beta}\right)$ for all $p_{i} \in P$ and $e_{i} \in Q \subseteq P \times P$.

Consider two cubic graphs $M_{1}=\left\langle\alpha_{1}, \beta_{1}\right\rangle$ and $M_{2}=\left\langle\alpha_{2}, \beta_{2}\right\rangle$ in $\Omega^{*}$. If we exchange $\mu_{\alpha_{1}}$ by $\mu_{\alpha_{2}}$ and $\mu_{\beta_{1}}$ by $\mu_{\beta_{2}}$ we get the cubic graph as $\widehat{M_{1}}=\left\langle\widehat{\alpha_{1}}, \widehat{\beta_{1}}\right\rangle$ and $\widehat{M_{2}}=\left\langle\widehat{\alpha_{2}}, \widehat{\beta_{2}}\right\rangle$, respectively.

For any two IC-graphs (or EC-graphs) $M_{1}$ and $M_{2}$, two cubic graphs $\widehat{M_{1}}$ and $\widehat{M}_{2}$ may not be IC-graph and EC-graph. 
Example 4.4. Consider two IC-graphs $M_{1}=\left\langle\alpha_{1}, \beta_{1}\right\rangle$ and $M_{2}=\left\langle\alpha_{2}, \beta_{2}\right\rangle$ as shown in figure 17 .

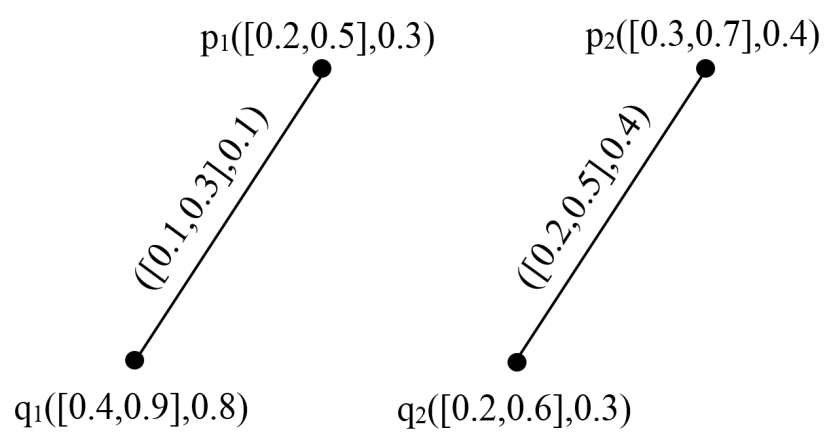

FiguRE 17. IC-graphs $M_{1}$ and $M_{2}$

Then, their corresponding $\widehat{M_{1}}$ and $\widehat{M}_{2}$ are shown in figure 18 .

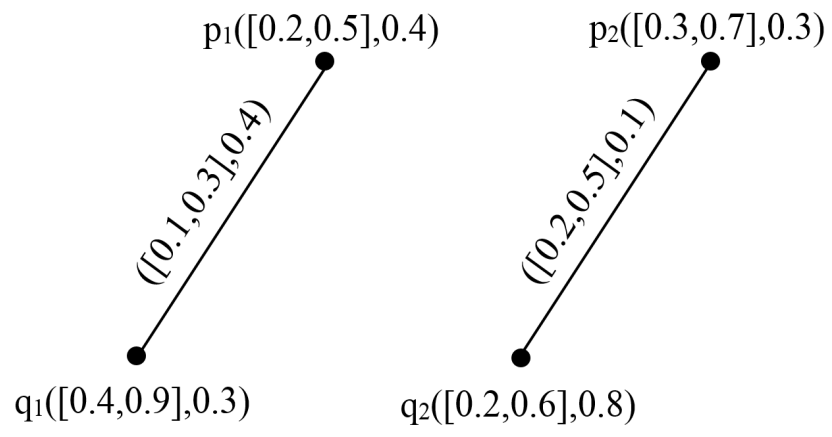

Figure 18. Cubic graphs $\widehat{M}_{1}$ and $\widehat{M}_{2}$

It is easy to see that the cubic graphs $\widehat{M}_{1}$ and $\widehat{M}_{2}$ are neither IC-graph nor EC-graph. Similarly, we can provide and example for two EC-graphs that are neither IC-graph nor EC-graph.

\section{Application}

Fuzzy graph theory is a platform which has wide range of applications in mathematics, computer science etc. Cubic graph is a more general approach, which can be used in decision making very effectively.

Suppose we have a set of three countries like, $P=\{X, Y, Z\}$ as a vertex set and the membership of each member of the set denotes the strength of that country over the neighbouring country with respect to future and present time by considering its economic strength. Now we want to observe the effect of strength of one country at the another country with respect to economy. Let we have a cubic set for each country based on 
certain information and data with respect to economy

$$
\alpha=\left\{\begin{array}{c}
\langle X:[0.6,0.8], 0.9\rangle \\
\langle Y:[0.5,0.9], 0.7\rangle \\
\langle Z:[0.3,0.7], 0.8\rangle
\end{array}\right.
$$

where interval membership predicts the economy of a certain country for the future and the other membership shows economy of a certain country for the present time based on certain information and data with respect to economy. Now on the basis of $\alpha$, we have the set $\beta$ of edges as follows

$$
\beta=\left\{\begin{array}{c}
\langle X Y:[0.5,0.8], 0.9\rangle \\
\langle Y Z:[0.3,0.7], 0.8\rangle \\
\langle Z X:[0.3,0.7], 0.9\rangle
\end{array}\right.
$$

where interval membership predicts the effect of economy of a certain country for the future and the other membership shows the effect of economy of a certain country for the present time at the other country. The corresponding cubic graph is shown in figure 19.

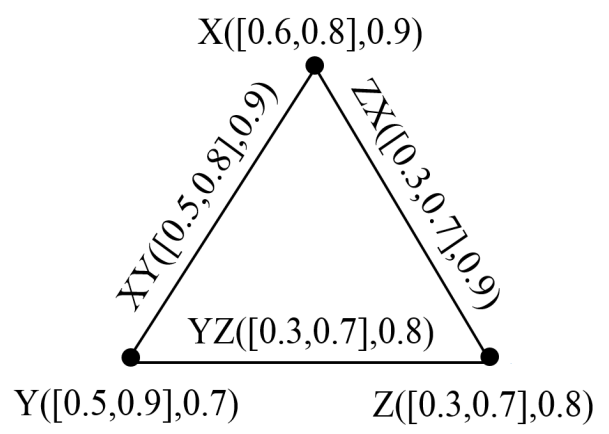

Figure 19. Cubic graph

So finally we concluded that economy of a certain country effect very much on the economy of the neighboring countries.

\section{Conclusions}

Graphs are among the most ubiquitous models of both natural and human-made structures. They can be used to model many types of relations and process dynamics in computer science, physical, biological and social systems. We come up here with the idea of cubic graphs and we define different operations of cubic graphs. We also provide a short application of cubic graph. In future we are planning to generalize our notions to (1) Cubic line graphs, (2) Cubic hypergraphs, and (3) Cubic soft graphs. 


\section{REFERENCES}

[1] M. Akram and W.A. Dudek, Interval-valued fuzzy graphs, Comput. Math. Appl., 61(2) (2011) 289-299.

[2] M. Akram, Interval-valued fuzzy line graphs, Neural Comput. Appl., 21 (2012) 145-150.

[3] M. Akram and B. Davvaz, Strong intuitionistic fuzzy graphs, Filomat, 26(1) (2012) 177-196

[4] M. Akram, Bipolar fuzzy graphs, Inf. Sci., 181 (2011) 5548-5564.

[5] M. Akram, Bipolar fuzzy graphs with applications, Knowl.based Syst., 39 (2013) 1-8.

[6] P. Bhattacharya, Some remarks on fuzzy graphs, Pattern Recognition Lett., 6 (1987) 297-302.

[7] R.A. Borzooei and H. Rashmanlou, Cayley interval-valued fuzzy threshold graphs, U.P.B. Sci. Bull., Ser. A, 78(3) (2016) 83-94.

[8] F. Buckley, Self-centered graphs, Ann. N.Y. Acad. Sci., 576 (1989) 71-78.

[9] Y.B. Jun, C.S. Kim and M.S. Kang, Cubic subalgebras and ideals of BCK/BCI-algebras, Far East J. Math. Sci., 44 (2010) 239-250.

[10] Y.B. Jun, K.J. Lee and M.S. Kang, Cubic structures applied to ideals of BCI-algebras, Comput. Math. Appl., 62(9) (2011) $3334-3342$.

[11] Y.B. Jun, G. Muhiuddin, M.A. Oztürk and E.H. Roh, Cubic soft ideals in BCK/BCI-algebras, J. Comput. Anal. Appl., $22(5)$ (2017) 929-940.

[12] Y.B. Jun, C.S. Kim and K.O. Yang, Cubic sets, Ann. Fuzzy Math. Inf., 4(1) (2012) 83-98.

[13] J.G. Kang and C.S. Kim, Mappings of cubic sets, Commun. Korean Math. Soc., 31(3) (2016) 423-431.

[14] A. Kauffman, Introduction a la Theorie des Sous-emsembles Flous, Masson et Cie, 1 (1973).

[15] M. Khan, Y.B. Jun, M. Gulistan, N. Yaqoob, The generalized version of Jun's cubic sets in semigroups, J. Intell. Fuzzy Syst., 28(2) (2015) 947-960.

[16] S.N. Mishra, H. Rashmanlou and A. Pal, Coherent category of interval-valued intuitionistic fuzzy graphs, J. Mult.-Val. Log. Soft Comput., 29(3-4) (2017) 355-372.

[17] J.N. Mordeson and P.S. Nair, Fuzzy graphs and fuzzy hypergraphs, Physica Verlag, Heidelberg (2001).

[18] G. Muhiuddin, S.S. Ahn, C.S. Kim and Y.B. Jun, Stable cubic sets, J. Comput. Anal. Appl., 23(5) (2017) $802-819$.

[19] M. Pal, S. Samanta and H. Rashmanlou, Some results on interval-valued fuzzy graphs, Int. J. Comput. Sci. Electr. Eng., 3(3) (2015) 2320-4028.

[20] R. Parvathi, M. G. Karunambigai and K. Atanassov, Operations on intuitionistic fuzzy graphs, Proc. IEEE Int. Conf. Fuzzy Syst., (2009) 1396-1401.

[21] T. Pramanik, M. Pal and S. Mondal, Interval-valued fuzzy threshold graph, Pac. Sci. Rev. A: Nat. Sci. Eng., 18(1) (2016) 66-71.

[22] T. Pramanik, S. Samanta and M. Pal, Interval-valued fuzzy planar graphs, International J. Mach. Learn. Cybern., 7(4) (2016) 653-664.

[23] A. Rosenfeld, Fuzzy graphs, Fuzzy sets and their applications, Academic Press, New York, (1975) 77-95.

[24] S. Sahoo and M. Pal, Product of intuitionistic fuzzy graphs and degree, J. Intell. Fuzzy Syst., 32(1) (2017) $1059-1067$.

[25] M.S. Sunitha and K. Sameena, Characterization of g-self centered fuzzy graphs, J. Fuzzy Math., 16 (2008) 787-791.

[26] S. Vijayabalaji and S. Sivaramakrishnan, A cubic set theoretical approach to linear space, Abstr. Appl. Anal., 2015 (2015) Article ID 5231298 pages.

[27] L.A. Zadeh, Fuzzy sets, Inf. Control, 8 (1965) 338-353. 